\title{
Relação entre gatekeeping materno e personalidade paterna em famílias com pré-escolares
}

\author{
Relación entre gatekeeping materno y personalidad paterna en familias \\ con pre-escolares \\ Relationship between Maternal Gatekeeping and Paternal Personality in \\ Families with Preschoolers
}

\author{
Larissa Paraventi \\ Carolina Duarte de Souza \\ João Paulo Koltermann \\ Mauro Luis Vieira \\ Universidade Federal de Santa Catarina
}

Doi: https://doi.org/10.12804/revistas.urosario.edu.co/apl/a.6164

\begin{abstract}
Resumo
O Gatekeeping materno é um fator importante a ser considerado para avaliar a proximidade e qualidade da relação do pai com os filhos. Este consiste em crenças e comportamentos maternos que podem facilitar ou dificultar o envolvimento paterno com a criança. Dentre vários fatores que afetam esses comportamentos maternos encontra-se a personalidade do pai. Como poucos estudos investigaram a relação entre estas variáveis, esta pesquisa objetivou comparar as percepções de pais e mães do gatekeeping materno e investigar os efeitos da percepção paterna de sua personalidade nas percepções materna e paterna do gatekeeping da mãe em famílias
\end{abstract}

com crianças de quatro a seis anos. Participaram 171 casais do Sul do Brasil que responderam ao Questionário de Gatekeeping Materno e Inventário dos Cinco Grandes Fatores de Personalidade. Os resultados apontaram níveis moderados de gatekeeping materno, e elevados níveis de conscienciosidade e amabilidade paterna. As análises comparativas indicaram que pais perceberam maiores níveis de confirmação da identidade materna em comparação às próprias mães, e as mães perceberam em maior nível que os pais que tarefas domésticas e cuidados com as crianças fazem parte do papel materno. A regressão linear múltipla indicou que quanto mais neuroticismo, maior foi a percepção paterna do controle

Larissa Paraventi ORCID ID: https://orcid.org/0000-0003-0825-8770

Carolina Duarte de Souza ORCID ID: https://orcid.org/0000-0003-3555-1120

João Paulo Koltermann ORCID ID: https://orcid.org/0000-0003-1279-8147

Mauro Luis Vieira ORCID ID: https://orcid.org/0000-0003-0541-4133

Dirigir correspondencia a Larissa Paraventi. Correio eletrônico: larissaparaventi@hotmail.com. Agradecemos às famílias pela participação na pesquisa, ao Conselho Nacional de Desenvolvimento Científico e Tecnológico (CNPq) e à Coordenação de Aperfeiçoamento de Pessoal de Nível Superior (CAPES) pelo apoio financeiro e aos demais integrantes da equipe de pesquisa envolvidos na coleta e tabulação dos dados.

Para citar este artigo: Paraventi, L., de Souza, C. D., Koltermann, J. P., \& Vieira, M. L. (2021). Relação entre gatekeeping materno e personalidade paterna em famílias com pré-escolares. Avances en Psicología Latinoamericana, 39(1), 1-15. https://doi.org/10.12804/ revistas.urosario.edu.co/apl/a.6164 
materno pelas normas e responsabilidades domésticas; e quanto mais extroversão, menos as mães relataram realizar o controle pelas normas e responsabilidades domésticas. Reforça-se a relação entre características pessoais em interação com construções histórico-sociais de gênero que afetam crenças e comportamentos maternos sobre tarefas domésticas e cuidados infantis, influenciando o envolvimento paterno.

Palavras-chave: maternal gatekeeping; personalidade; paternidade; desenvolvimento; família.

\section{Resumen}

El gatekeeping materno es considerado un factor importante para evaluar la cercanía y la calidad de la relación del padre con sus hijos. Se trata de creencias y comportamientos maternos que pueden facilitar o dificultar la participación paterna con el niño. Entre varios factores que afectan estos comportamientos maternos se encuentra la personalidad del padre. Como pocos estudios han investigado la relación entre estas variables, esta investigación tuvo como objetivo comparar las percepciones de padres y madres sobre el gatekeeping materno e investigar los efectos de la percepción paterna de su personalidad en las percepciones maternas y paternas sobre el gatekeeping materno en familias con hijos de cuatro a seis años. En el estudio participaron 171 parejas del sur de Brasil que respondieron el Cuestionario de Gatekeeping materno y el Inventario de los Cinco Grandes Factores de Personalidad. Los resultados mostraron niveles moderados de gatekeeping materno, altos niveles de escrupulosidad y amabilidad paterna. Los análisis comparativos indicaron que los padres percibieron niveles más altos de confirmación de la identidad materna en comparación con las propias madres, y las madres percibieron a un nivel más alto que los padres, que las tareas del hogar y el cuidado de los niños son parte del rol materno. La regresión lineal múltiple indicó que cuanto más neuroticismo, mayor era la percepción paterna del control materno por las normas y responsabilidades domésticas; y cuanto más extroversión, menos madres informaron tener control sobre las normas y responsabilidades domésticas. Se refuerza la relación entre características personales en interacción con construcciones histórico-sociales de género que inciden en las creencias y comportamientos maternos sobre las tareas domésticas y el cuidado de los niños, incidiendo en la participación paterna.

Palabras clave: maternal gatekeeping; personalidad; paternidad; desarrollo; familia.

\section{flbstract}

Maternal Gatekeeping is an important factor to consider when assessing the closeness and quality of the father's relationship with his children. It consists of maternal beliefs and behaviors that can facilitate or hinder the paternal involvement with the child. Among several factors that affect these maternal behaviors is the father's personality. As few studies have investigated the relationship between these variables. This research was aimed at comparing fathers' and mothers' perceptions of maternal gatekeeping and investigate the effects of paternal perception of their personality on maternal and paternal perceptions of mother's gatekeeping in families with children aged four to six years old. A total of 171 couples from southern Brazil who answered the Maternal Gatekeeping Questionnaire and the Big Five Personality Factors Inventory participated in the study. The results showed moderate levels of maternal gatekeeping and high levels of conscientiousness and paternal kindness. Comparative analyzes indicated that fathers perceived higher levels of confirmation of maternal identity compared to mothers themselves, and mothers perceived at a higher level than fathers that household chores and childcare are part of the maternal role. Multiple linear regressions indicated that the more neuroticism, the greater was the paternal perception of maternal control by norms and domestic responsibilities, and the more extroversion, the fewer mothers reported having control over domestic norms and responsibilities. The relationship between personal characteristics in interaction with historical-social constructions of gender that affect maternal beliefs and behaviors about household chores and childcare, influencing paternal involvement is reinforced.

Keywords: Maternal gatekeeping; personality; paternity; development; family. 
O desenvolvimento infantil é influenciado por um complexo sistema de interações, dentre as quais se destacam o relacionamento mãe-pai-criança por sua relevância para os efeitos socioemocionais, cognitivos, neurobiológicos e de saúde infantis. Por este motivo pesquisar sobre o papel que pais, criança e fatores contextuais exercem na promoção do desenvolvimento infantil e manutenção de uma relação saudável entre pais-criança torna-se relevante (Frosch et al., 2021). A maioria dos estudos sobre a relação pais-filhos ainda se foca apenas na mãe (Cabrera \& Volling, 2019), apesar de uma análise retrospectiva de pesquisas sobre o envolvimento de pais (homens) com seus/ suas filhos/as indicar que o envolvimento paterno ao longo do ciclo vital é preditor de diferentes esferas desenvolvimentais dos/as filhos/as (Volling \& Cabrera, 2019).

O envolvimento do pai com o filho(a) depende de variáveis sociais e ambientais, bem como de variáveis individuais dos pais e das crianças, as quais atuam sobre e são influenciadas umas pelas outras (Volling \& Cabrera, 2019). Um aspecto específico que tem sido apontado por pesquisas como fator de importante influência para a qualidade e proximidade da relação pai-filho/a é o gatekeeping materno (Cannon et al., 2008; Hauser, 2012; Piskernik \& Ahnert, 2019; Schoppe-Sullivan et al., 2015; Zvara et al., 2013).

O gatekeeping materno é um fenômeno relativo à regulação materna do envolvimento do pai com os filhos. Originalmente ele se referia às crenças e comportamentos maternos que inibem o envolvimento paterno com o trabalho doméstico e o cuidado com os(as) filhos(as) (Allen \& Hawkins, 1999). Esse modelo pioneiro engloba três dimensões do fenômeno: (1) Normas e responsabilidades: diz respeito aos esforços maternos em manter as responsabilidades pelas tarefas de casa e cuidados com os filhos para si e ao controle do estabelecimento das normas, responsabilidades e padrões de como as tarefas devem ser desempenhadas; (2) Confirmação de identidade materna: refere-se ao quanto a mãe associa sua própria identidade como mãe baseando-se na sua competência com as tarefas de casa, com o cuidado dos filhos, validados por outras pessoas; e (3) Papéis familiares diferenciados: corresponde às expectativas das mulheres sobre as capacidades $\mathrm{e}$ a pró-atividade dos homens para fazerem as tarefas de casa e cuidarem dos filhos que reforçam a crença de diferenciação entre papéis paterno e materno, em que o trabalho doméstico e familiar é exaltado como função exclusiva materna.

Essas dimensões e o conceito em si estão atrelados ao pressuposto cultural de gênero que atribui às mulheres o encargo das funções relativas ao espaço doméstico e cuidado das crianças (Allen \& Hawkins, 1999; Zvara et al., 2013). Dessa forma, pesquisas apontam que mães que percebem os afazeres domésticos e a criação dos filhos como encargos femininos tendem a exercer maior controle sobre o envolvimento do pai com os filhos (Cannon et al., 2008), o que reforça a estereotipia de papéis tradicionais de gênero (Allen \& Hawkins, 1999).

Compreende-se gênero em uma perspectiva socioconstrucionista que parte dos sentidos criados por meio de (re)significações acerca dos corpos, que por sua vez são elaboradas em interações permeadas por posições de poder (Nogueira, 2001). Desse modo, compreende-se que diferenças no envolvimento paterno e materno são atravessadas por aprendizagens realizadas por décadas de distinções socioculturais de gênero de modos de ser pai e ser mãe (Palkovitz et al., 2014).

Apesar dessa conceituação pioneira do gatekeeping materno ainda ser empregada em estudos mais recentes (Holmes et al., 2013; Sweeney et al., 2017), atualmente existem duas outras conceituações contemporâneas que abarcam, além dos aspectos negativos, características positivas do fenômeno. Puhlman e Pasley (2017) realizaram uma revisão teórica do conceito e propuseram outras três dimensões: (a) restritiva (ações maternas que desencorajam o envolvimento paterno), (b) facilitadora (encorajamento materno do envolvimento 
do pai com os/as filhos/as) e (c) controle (o quanto a mãe divide —ou não- as responsabilidades de tomada de decisão dos assuntos familiares com a dupla coparental). Ainda há uma subdivisão do conceito em maternal gate closing que engloba comportamentos e crenças maternos desencorajadores (criticar a parentalidade paterna, refazer as atividades domésticas realizadas pelo pai, controlar as decisões acerca da criação dos filhos) e maternal gate opening que engloba comportamentos da mãe encorajadores do envolvimento do pai com os/as filhos/as (considerar a opinião paterna em assuntos relativos à parentalidade, organizar atividades para o pai realizar com a criança) (Lee et al., 2019; Schoppe-Sullivan et al., 2008; Schoppe-Sullivan et al., 2015).

No momento de concepção e coleta de dados do presente estudo, os pesquisadores não tiveram acesso a instrumentos de medida do gatekeeping materno que abordassem seus aspectos positivos, o que acarretou na opção por utilizar o modelo de Allen e Hawkins (1999) como referência. Entretanto, compreende-se o fenômeno de forma mais ampla, de acordo com estudos mais recentes, sobre como os pressupostos culturais de gênero sobre a parentalidade determinam o comportamento materno de permitir ou impossibilitar que o pai se envolva nos cuidados com seus/suas filhos/as (Fagan \& Cherson, 2017; Puhlman \& Pasley, 2017).

As pesquisas sugerem que o gatekeeping materno é um fenômeno multideterminado que sofre influências de características pessoais (da mãe, do pai e da criança), relacionais e contextuais (Allen \& Hawkins, 1999; Schoppe-Sullivan et al., 2015). Dentre essas influências o funcionamento psicológico tem sido pouco investigado pela literatura (Schoppe-Sullivan et al., 2015), o que inclui a personalidade de mães e pais. A carência de estudos que busquem compreender essa relação pode ser reforçada pela constatação da meta-análise de 30 estudos realizada por Prinzie et al. (2009) de que a personalidade (investigada pelo modelo dos Cinco Grandes Fatores - CGF-) é uma característica interna relevante que afeta os aspectos relacionados à parentalidade. Isso por compreender que o gatekeeping materno impacta e tem relação com a parentalidade de pais e mães (Fagan, 2020), o que reforça a relevância de investigar a relação entre gatekeeping materno e personalidade.

A maioria dos estudos sobre personalidade tem utilizado o modelo dos Cinco Grandes Fatores (CGF) da personalidade, o qual trabalha os traços de personalidade como representações de tendências básicas de um indivíduo que em interação com o ambiente, produzem competências, crenças, atitudes as quais influenciam as relações interpessoais, para compreender e acessar o fenômeno (De Young et al., 2007). E de acordo com Prinzie et al. (2009), apesar da existência de críticas a esse modelo, ele tem se mostrado uma ferramenta útil na conceitualização e mensuração da personalidade, assim como na sistematização e comparação entre as pesquisas. Esse modelo classifica os traços de personalidade em cinco domínios: (1) Extroversão: diz respeito à quantidade e à intensidade das interações interpessoais. Maior extroversão está relacionada a ser sociável, dominante, enérgico, ativo, expressivo e capaz de expressar emoções positivas, como alegrar-se; (2) Amabilidade: refere-se às características de relacionamento interpessoal direcionadas aos outros, altos níveis neste traço estão relacionados a características como afetuosidade, cooperação, altruísmo, amabilidade, agradabilidade; (3) Conscienciosidade: representa o grau de organização, persistência, controle e motivação para alcançar objetivos. Altos níveis neste traço estão relacionados a pessoas responsáveis, confiáveis, organizadas, capazes de controlar impulsos e com facilidade para executar obrigações; (4) Neuroticismo: é o fator que mais abrange as características emocionais, refere-se a níveis contínuos de instabilidade e ajustamento emocional. Altos níveis neste traço indicam pessoas nervosas, altamente sensíveis, tensas, preocupadas, irritadiças, melancólicas, envergonhadas, instáveis emocionalmente; (5) Abertura: refere-se a comportamentos exploratórios, busca por 
novas soluções e reconhecimento da importância de ter novas experiências. Altos níveis neste traço representam pessoas francas, imaginativas, espirituosas, originais, com uma mente complexa, aberta e profunda (Andrade, 2008).

$\mathrm{Na}$ revisão para a presente pesquisa identificaram-se apenas quatro estudos que focaram na relação entre personalidade e gatekeeping materno, dentre os quais três investigaram a personalidade do pai e da mãe, e somente um acessou aspectos positivos e negativos da personalidade por meio do CGF, e apenas um avaliou o poder preditivo de aspectos do funcionamento psicológico e personalidade para o gatekeeping materno. Cannon et al. (2008) investigaram a relação entre um aspecto negativo e um positivo das personalidades materna e paterna e sua relação com o gatekeeping materno em aspectos de gate closing e gate opening e seus resultados indicaram uma correlação negativa entre a presença de emocionalidade negativa paterna (medo e ansiedade) e gate opening da mãe. A pesquisa também identificou que as crenças parentais sobre o papel paterno na criação dos filhos (tradicionais - pai provedor; ou progressista - pai envolvido emocionalmente e em cuidados básicos) exercem um papel moderador na relação entre aspectos negativos da personalidade de pais e mães e os comportamentos maternos de gate closing, de modo que quando pais/mãe possuíam esse traço de personalidade negativo, mas suas crenças em relação ao papel paterno eram progressivos, esses atuavam de modo a inibir a presença de comportamentos negativos de gatekeeping materno.

A pesquisa de Lee (2010), a única a acessar personalidade por meio do CGF, investigou apenas a mãe e encontrou correlações negativas entre aspectos gate closing do gatekeeping materno e aspectos positivos de personalidade materna (amabilidade), e correlações positivas com aspectos negativos de personalidade materna (neuroticismo), além de uma correlação positiva entre conscienciosidade e desejo materno de controle das decisões parentais. Ainda se correlacionaram positivamente os aspectos gate opening do gatekeeping materno com características maternas de extroversão. O estudo de Sweeney et al. (2017) investigou a relação entre gatekeeping e neuroticismo, mas não encontrou correlações tanto para pais quanto para mães em casais heterossexuais e homoafetivos (femininos e masculinos).

Por fim, os resultados de Schoppe-Sullivan et al. (2015) indicaram correlações positivas entre aspectos gate closing do gatekeeping materno com aspectos negativos do funcionamento psicológico e personalidade materna (neuroticismo, ansiedade e depressão), e não foram encontradas correlações entre gatekeeping materno e aspectos da personalidade paterna. Este foi o único estudo a avaliar fatores psicológicos individuais como possíveis preditores do gatekeeping materno, os resultados apontaram que um funcionamento psicológico empobrecido foi preditor de maiores relatos de gate closing pelas mães, mas não houve predição em relação ao gate opening.

Dessa forma, dos quatro estudos que investigaram a relação entre os fenômenos, três encontraram relações estatisticamente significativas, o que indica a necessidade de mais pesquisas para compreender a relação entre gatekeeping materno e personalidade. Com vistas a contribuir com esse campo de estudo a presente pesquisa objetivou comparar as percepções de pais e mães do gatekeeping materno e investigar os efeitos da percepção paterna de sua personalidade por meio do CGF nas percepções materna e paterna do gatekeeping da mãe em famílias com crianças de quatro a seis anos. Delimitou-se a faixa etária com o intuito de levantar dados sobre as fases iniciais do desenvolvimento infantil, quando ainda há grande demanda por realização de tarefas domésticas; menor dependência de cuidados em sua maioria exclusivos maternos, como a amamentação; e por ser um período sensível do desenvolvimento infantil para socialização, em que a criança passa a interagir mais com a figura paterna (Bossardi et al., 2013).

Esta pesquisa torna-se mais relevante à medida que nenhum estudo sobre gatekeeping materno 
como construto principal foi identificado no Brasil, e somente três estudos internacionais que o correlacionaram com a personalidade paterna foram identificados. Primeiramente hipotetiza-se que mães e pais tenham percepções diferentes do gatekeeping materno: no caso das dimensões de normas e responsabilidades e confirmação da identidade materna espera-se que os índices paternos sejam maiores, mas que os índices maternos sejam maiores em papéis familiares diferenciados. Hipotetiza-se que a personalidade paterna é preditora do gatekeeping materno. Ainda, hipotetiza-se que a predição dos quatro aspectos desejáveis da personalidade do pai (extroversão, amabilidade, conscienciosidade e abertura) é negativa para todas as dimensões do gatekeeping materno (normas e responsabilidades, confirmação da identidade materna e papéis familiares diferenciados) e que a predição do neuroticismo paterno é positiva para as dimensões do gatekeeping materno.

\section{Método}

\section{Delineamento e participantes}

Estudo empírico do tipo transversal com intuito de caracterizar o gatekeeping materno e a personalidade do pai e suas correlações. O levantamento de dados ocorreu por autorrelato por meio de questionários aplicados com os participantes. Quanto aos objetivos, teve caráter exploratório e descritivo. Fizeram parte da pesquisa 171 casais heteroafetivos (ou seja, 171 pais/padrastos e 171 mães/madrastas) com filhos em idades entre 4 e 6 anos. Mantinham residência em três estados do sul do Brasil: Santa Catarina ( $\mathrm{N}=126)$, Paraná $(\mathrm{N}=30)$ e Rio Grande do Sul $(\mathrm{N}=15)$, escolhidos por conveniência. Foram incluídas famílias biparentais que tivessem pelo menos um(a) filho(a), biológico(a) ou não, em idade pré-escolar que deveria ter nascido posteriormente aos 18 anos de idade dos pais, e o casal deveria coabitar há pelo menos seis meses.
A média de idade das mães foi 35 anos $(D P=5.79)$ e dos pais 38 anos $(D P=6.77)$. A maioria dos casais participantes $(80.4 \%)$ constituía família nuclear sendo pais biológicos de todos os filhos, sendo que famílias recasadas com pais biológicos da criança alvo $(9.2 \%)$ apareceu em segundo lugar. A média dos anos de escolaridade para mãe foi 16 anos $(D P=5.87)$ e para o pai foi 15 anos $(D P=5.76)$. No que diz respeito à participação no mercado de trabalho, $9.5 \%$ das mulheres relatou não possuir jornada de trabalho fora de casa, ao passo que $4.8 \%$ dos homens também o relataram, sendo classificados como desempregados ou trabalhadores domésticos. Constatou-se que a jornada de trabalho de 40 horas semanais foi a mais frequente, sendo relatada por $38.1 \%$ das mulheres e $54.8 \%$ dos homens. Em termos de horas de trabalho, a média da jornada de trabalho feminina foi 34 horas $(D P=14.07)$ e a masculina foi 40 horas $(D P=13.31)$.

\section{Instrumentos}

Questionário Sociodemográfico: composto por 18 questões referentes a variáveis como cidade de residência, composição familiar, idade, escolaridade, jornada de trabalho e presença de funcionário contratado para auxiliar nos cuidados com a casa e com a(s) criança(s). Tal instrumento foi aplicado exclusivamente com as mães.

Inventário dos Cinco Grandes Fatores de Personalidade (Big Five): elaborado originalmente por John et al. (1991) composto por 44 itens. Tem o objetivo de avaliar a personalidade a partir do modelo dos Cinco Grandes Fatores, segundo as dimensões de extroversão (aconchegante, agregador, ativo, assertivo, comunicativo), amabilidade (confiante, altruísta, modesta, tímida), conscienciosidade (organização, persistência, disciplina), neuroticismo (ansiedade, hostilidade, insegurança, depressão) e abertura (curiosidade, interesses artísticos, imaginação, perspicaz) (McCare \& John, 1992). Neste estudo, utilizou-se a versão validada para o Brasil, por Andrade (2008) que possui 
32 itens. São respondidos em uma escala tipo Likert de cinco pontos, a qual indica o nível de identificação das pessoas com características descritas nos itens variando de 1 "discordo totalmente" a 5 "concordo totalmente". No presente estudo, para verificar as propriedades psicométricas do inventário para amostra estudada optou-se por realizar a avaliação fatorial por meio de Análise Fatorial Confirmatória para dados ordinais com auxílio do pacote Lavaan, versão 0.5-22 (Rosseel, 2012) disponível para a linguagem de programação R, versão 3.1. Verificou-se a presença de cargas fatoriais cruzadas em dois itens: 37 ("Gera muito entusiasmo"), com cargas fatoriais em Abertura e Amabilidade; e o item 29 ("É cheio de energia") com cargas em Amabilidade, Conscienciosidade e Abertura. Para manter o caráter congenérico da escala, optamos pela remoção desses dois itens pertencentes originalmente ao fator de extroversão. A fidedignidade foi avaliada por meio do alfa de Cronbach cujos valores foram: 0.81 para Abertura; 0.74 para Conscienciosidade; 0.79 para Extroversão; 0.81 para Amabilidade; e 0.79 para Neuroticismo. Este inventário foi aplicado somente com os pais.

Questionário de Gatekeeping Materno (GTK): originalmente desenvolvido por Allen e Hawkins (1999). Objetiva avaliar o gatekeeping materno por meio de três dimensões: (1) Normas e responsabilidades: diz respeito a quanto a mãe se esforça para manter as responsabilidades pelas tarefas de casa ao controlar o estabelecimento de normas; (2) Confirmação de identidade materna: refere-se ao quanto a mãe associa sua própria identidade como mãe com sua competência nas tarefas de casa; e (3) Papéis familiares diferenciados: corresponde às expectativas e crenças das mulheres sobre a capacidade e pró-atividade dos homens para fazer as tarefas de casa. É composto por 11 itens, respondidos numa escala de quatro pontos para indicar o quanto descreve a pessoa, variando de 1 "não me/a descreve" até 4 "me/a descreve muito bem".

O instrumento original é respondido pelas mães e apresentou coeficientes de fidedignidade variando de 0.66 a 0.79 (Allen \& Hawkins, 1999). Para a presente pesquisa adequou-se o GTK ao português e à população brasileira, tendo em vista que o instrumento não apresentava validação para o Brasil. O GTK foi adaptado também para que mães e pais respondessem aos mesmos itens sobre os comportamentos e crenças maternas, pois se considerou que a percepção paterna sobre o papel da mãe na família é outra fonte relevante de investigação do gatekeeping materno. $\mathrm{O}$ instrumento apresentou os seguintes valores de precisão para pais e mães respectivamente: Normas e responsabilidades $(\alpha=0.77 / \alpha=0.79)$; Confirmação da identidade materna $(\alpha=0.46 / \alpha=0.49)$ e Papéis familiares diferenciados $(\alpha=0.63 / \alpha=0.70)$.

\section{Procedimentos de coletas de dados}

As famílias foram acessadas por meio de instituições de educação infantil, escolhidas por acessibilidade, bem como por "bola de neve", por meio da qual as famílias que participaram da pesquisa, indicaram outras famílias como possíveis participantes. A coleta de dados foi realizada no domicílio da família ou na UFSC após assinatura do termo de consentimento livre e esclarecido (TCLE).

A pesquisa atendeu aos parâmetros éticos seguindo a resolução 466, de 12 de dezembro de 2012 do Conselho Nacional de Saúde. Fez parte do projeto "Relações entre envolvimento e práticas parentais, funcionamento familiar, coparentalidade e comportamento da criança pré-escolar", aprovado pelo Comitê de Ética em Pesquisa com Seres Humanos da UFSC sob o número 447.932.

\section{Procedimento de análise dos dados}

Os dados obtidos foram tabulados e submetidos a análises estatísticas por meio da utilização do pacote estatístico Statistical Package for Social Sciences (SPSS) - versão 22.0. Utilizou-se estatísticas descritivas para caracterizar a amostra e as variáveis de interesse e as seguintes análises 
inferenciais: teste $\mathrm{T}$ para amostras pareadas para comparação da percepção de pais e mães do gatekeeping materno; regressões múltiplas com nível de percepção das dimensões de gatekeeping materno de pais e mães como desfecho e as dimensões da personalidade paterna como preditoras.

\section{Resultados}

\section{Caracterização e comparação do gatekeeping materno segundo os participantes}

A análise estatística indicou que as três dimensões do gatekeeping materno apresentaram médias muito próximas ao centro da escala como pode ser observado na tabela 1. Os dados apresentados na tabela 1 demonstram que pais e mães relataram percepções medianas de gatekeeping materno, posto que todas as médias variaram entre $2.08 \mathrm{e}$ 2.56. Ao considerar que os escores do instrumento variam entre um e quatro, e que quatro era o máximo, pode-se afirmar que mães e pais tiveram pontuações médias em todas as dimensões. De modo que pais relataram maior percepção de Confirmação da Identidade Materna, e mães perceberam mais Papéis Familiares Diferenciados. Ao passo que para os pais a percepção de Papéis Familiares Diferenciados foi a menor média e para as mães foi a dimensão de Normas e Responsabilidades.

A tabela 1 ainda apresenta os resultados dos testes estatísticos para diferença de médias entre pais e mães. Apenas as médias da dimensão Normas e Responsabilidades não apresentaram diferença estatisticamente significativa, com diferença mínima na percepção dos cuidadores. Indicativo de que os cuidadores parecem concordar com a ocorrência mediana de esforços maternos para manter as responsabilidades pelas tarefas de casa ao controlar o estabelecimento de normas. Na dimensão de Confirmação da Identidade Materna a diferença estatisticamente significativa apontou que os pais indicaram que as mães associam sua própria identidade como mães com sua competência nas tarefas de casa mais que elas mesmas. Ao passo que a diferenças estatisticamente significativa na dimensão de $\mathrm{Pa}$ péis familiares diferenciados indicou que as mães têm maiores expectativas e crenças que os pais de que homens não são capazes nem proativos para fazer as tarefas de casa e cuidar dos filhos, as mães possuem crenças e expectativas maiores sobre a capacidade e pró-atividade dos homens para fazer as tarefas de casa.

Tabela 1.

Médias, desvios padrões e comparações da percepção das dimensões de gatekeeping materno de pais e mães

\begin{tabular}{|c|c|c|c|c|}
\hline Dimensão & Cuidador & M (DP) & $\mathrm{t}$ & $\mathrm{p}$ \\
\hline \multirow{2}{*}{ Normas e responsabilidades } & Mãe & $2.12(0.75)$ & \multirow{2}{*}{$0.253(170)$} & \multirow{2}{*}{0.80} \\
\hline & Pai & $2.11(0.76)$ & & \\
\hline \multirow{2}{*}{ Confirmação de identidade } & Mãe & $2.35(0.65)$ & \multirow{2}{*}{$-3.949(170)$} & \multirow{2}{*}{$0.00^{*}$} \\
\hline & Pai & $2.56(0.72)$ & & \\
\hline \multirow{2}{*}{ Papéis familiares diferenciados } & Mãe & $2.46(0.83)$ & \multirow{2}{*}{$4.992(170)$} & \multirow{2}{*}{$0.00 *$} \\
\hline & Pai & $2.08(0.80)$ & & \\
\hline
\end{tabular}

Nota: $\mathrm{M}=$ média; $D P=$ desvio padrão; $t=$ teste $t ; p=$ teste de significância.

$* p<0.001$. 


\section{Caracterização da personalidade do pai segundo os participantes}

Os pais apontaram médias maiores que o centro da escala (3) em todas as dimensões exceto neuroticismo que indicou média abaixo do centro da escala. A dimensão com maior escore foi conscienciosidade $(\mathrm{M}=4.51 ; D P=0 ., 82)$, indicando que os pais se consideram organizados, persistentes, controlados e motivados para alcançar objetivos. Com uma média também alta de amabilidade $(M=4.37$; $D P=0.58$ ), indica que os pais se perceberam descontraídos, responsivos, empáticos às necessidades dos outros. No que tange à dimensão abertura, a média de escore foi $3.77(D P=0.58)$. Desse modo, os pais relataram que exibem comportamentos exploratórios e que reconhecem a importância de se ter novas experiências. Já a dimensão extroversão, a média de $3.26(D P=0.78)$ aponta que os pais estabelecem interações interpessoais, realizam atividade, necessitam de estimulação e têm a capacidade de alegrar-se de maneira moderada. Por fim, a dimensão com menor escore foi neuroticismo $(M=2.69 ; D P=0.82)$, destacando que os pais acreditam romper com maior frequência os níveis contínuos de instabilidade e mostraram-se mais ajustados emocionalmente.

\section{Predição da personalidade paterna para a percepção de gatekeeping materno de mães e pais}

As análises de regressão múltiplas realizadas entre os cinco fatores de personalidade do pai predizendo as dimensões das percepções de gatekeeping materno das mães e dos pais, apontaram a existência de relações estatisticamente significativas apenas nas dimensões Normas e Responsabilidades, tanto para pais como para mães. Como pode ser observado na tabela 2, para as mães, quanto mais o pai referiu ser extrovertido menos as mães relataram realizar o controle no estabelecimento de normas e responsabilidades pelos afazeres domésticos. De modo que ocorreu um efeito negativo da extroversão paterna sobre o gatekeeping materno de Normas e Responsabilidades. Entretanto, quanto mais os pais relataram características de neuroticismo, maior foi a percepção deles do controle materno pelas normas e responsabilidades domésticas.

\section{Discussão}

O objetivo do presente estudo foi comparar as percepções de pais e mães do gatekeeping materno, e investigar os efeitos da percepção paterna de sua personalidade por meio do CGF nas percepções materna e paterna do gatekeeping da mãe, em famílias com crianças de quatro a seis anos. As hipóteses iniciais referentes à existência de percepções diferentes do gatekeeping materno para mães e pais e a personalidade como preditora do gatekeeping materno foram ambas parcialmente confirmadas. Assim, este estudo identificou a personalidade paterna como fator determinante do gatekeeping materno, cuja influência na qualidade e quantidade da relação pai-criança, implicando em benefícios e/ou prejuízos ao desenvolvimento infantil, vem ganhando destaque na literatura científica (Cannon et al., 2008; Hauser, 2012; Piskernik \& Ahnert, 2019; Schoppe-Sullivan et al., 2015; Zvara et al., 2013).

O reconhecimento nessa amostra, da existência de níveis intermediários de gatekeeping materno por pais e mães e da relação desses comportamentos com traços de personalidade paterna, apontam para uma transformação cultural em curso sobre as relações de gênero e, por conseguinte dos papéis esperados/desempenhados por pais e mães no contexto do Sul do Brasil. Os comportamentos de gatekeeping materno aqui mensurados, se relacionam a uma construção histórica e social de gênero sobre os papéis maternos e paternos distintos, sem divisão igualitária de tarefas. É possível pensar que o resultado encontrado pode ser ainda resultado 
Tabela 2.

Regressões múltiplas significativas entre as dimensões da Personalidade Paterna e as percepções de pai e mãe sobre as dimensões de gatekeeping materno

\begin{tabular}{|c|c|c|c|}
\hline Participante & Dimensão de personalidade paterna & Dimensão do Gatekeeping materno & $\begin{array}{c}\text { Coeficiente de regressão } \\
\text { padronizado } \beta\end{array}$ \\
\hline \multirow{5}{*}{ Pai } & Abertura & \multirow{5}{*}{ Normas e responsabilidades } & 0.11 \\
\hline & Consienciosidade & & -0.12 \\
\hline & Amabilidade & & 0.11 \\
\hline & Neuroticismo & & $0.18^{*}$ \\
\hline & Extroversão & & -0.07 \\
\hline \multirow{5}{*}{ Mãe } & Abertura & \multirow{5}{*}{ Normas e responsabilidades } & 0.03 \\
\hline & Consienciosidade & & -0.14 \\
\hline & Amabilidade & & 0.11 \\
\hline & Neuroticismo & & 0.06 \\
\hline & Extroversão & & $-0.16^{*}$ \\
\hline
\end{tabular}

Nota: ${ }^{*} \mathrm{p} \leq 0.05 .{ }^{*} \mathrm{p} \leq 0.01$.

desses atravessamentos socioculturais de gênero nos modos de ser pai e mãe, tendo em vista que parte da identidade materna e paterna se formam com base nos reforçamentos culturais do que é esperado sobre o comportamento materno e paterno, e parte pelas lembranças de práticas parentais da infância dos próprios pais (Palkovitz et al., 2014).

Portanto, mães e pais perceberam uma prática moderada realizada pelas mães de gatekeeping materno, com comportamentos maternos que ainda corroboram barreiras culturais para uma corresponsabilidade parental das atividades domésticas e criação dos filhos. Resultados que vão ao encontro da pesquisa de Allen e Hawkins (1999) na qual $42 \%$ das mães foram classificadas em um cluster intermediário com níveis medianos de comportamentos e crenças maternas que influenciam no envolvimento do pai com atividades relacionadas ao trabalho doméstico e ao cuidado com os filhos. Tal resultado, que foi pouco explorado pelas autoras à época, a ser retomado a partir de perspectivas mais contemporâneas do fenômeno, pode ser analisado paralelamente à dimensão controle proposta por Puhlman e Pasley (2017) que permite investigar o quanto as mães tomam o lugar de decisão das responsabilidades familiares sem incluir o pai na tomada de decisão. Quando os níveis dessa dimensão de controle são baixos as mães tendem a abrir espaço para estabelecerem papéis mais igualitários com os parceiros, não se comportam como supervisoras ou ditam como deve ser o envolvimento do pai com os filhos, e contrariamente ao estereótipo paterno atualmente veiculado, confiam nas habilidades parentais paternas (Puhlman \& Pasley, 2017).

No que diz respeito a tais considerações, vale ressaltar que o destaque de um nível intermediário de gatekeeping materno percebido por mães e pais representa uma manutenção de um ambiente mais flexível entre os parceiros, no qual é possível que ambos contribuam para os mais variados domínios da vida familiar, ainda que a mãe não confie ou 
implique os pais na execução de algumas tarefas. Ao permitirem essa troca de papéis familiares no cuidado diário com os filhos e na realização das tarefas domésticas, o casal potencializa seu empenho em proteger, promover e fomentar um crescimento saudável para seus filhos (Volling \& Cabrera, 2019). Em contraposição a indicações de níveis extremados de gatekeeping materno, que poderiam contribuir tanto para um bloqueio ou desencorajamento do envolvimento paterno caso os índices fossem extremamente elevados (Cannon et al., 2008; Piskernik \& Ahnert, 2019; Puhlman \& Pasley, 2017; Schoppe-Sullivan et al., 2015; Zvara et al., 2013).

A percepção de pais e mães na dimensão de "normas e responsabilidades" refutou a hipótese de diferença nas percepções. A similaridade das respostas paternas e materna com escore mediano aponta para a diminuição das crenças de que as mães são responsáveis pelos afazeres domésticos e pelo resultado do gerenciamento familiar dessas atividades. O que não impede que apresentem comportamentos de restrição ou facilitação, porém as limitações do instrumento de pesquisa não permitiram acesso a esse dado que seria importante para compor um perfil de gatekeeping para essa amostra a partir das três dimensões propostas por Puhlman e Pasley (2017). Esse resultado aponta para uma mudança em relação à pesquisa de Hauser (2012), que também tinha como fonte de informação o casal, a qual apontava que as mães relataram com frequência um esforço para manter para si as responsabilidades, pois acreditavam que os parceiros não são tão bons quanto elas na realização das tarefas domésticas, e os pais nos relatos reforçavam esse ponto de vista.

Mães e pais apresentaram percepções diferentes do gatekeeping materno em duas dimensões, confirmando parcialmente essa hipótese inicial. Em relação à confirmação da identidade materna, os índices paternos foram maiores que os maternos. Isso aponta para a diferença das transformações culturais entre os gêneros. Pode-se notar que mães atrelaram menos sua identidade materna a afaze- res domésticos, cuidado com os filhos e validação de outras pessoas do que a percepção que os pais têm desses comportamentos da mãe. Parte desse resultado pode indicar que, ao refletir sobre o assunto, por desejabilidade social, as mães sintam vergonha em afirmar que precisam dessa validação, entretanto também é possível que em uma sociedade patriarcal os homens demorem mais a perceber mudanças que impliquem em perdas de privilégios, e acabem por reforçar essa dimensão do gatekeeping materno.

Essa reflexão estende-se para a diferença de percepção parental da dimensão de Papéis familiares diferenciados, em que as mães reportaram médias maiores que as paternas. Isso porque parte da concordância materna com as crenças de incapacidade masculina para realizar tarefas de casa e cuidarem dos filhos, pode ser decorrente da percepção da estrutura patriarcal e não necessariamente de uma diferenciação essencializada entre homens e mulheres. Isso porque de fato os homens que hoje exercem a parentalidade, raramente foram educados para exercerem uma parentalidade ativa com cuidado e afeto, mas sim para serem um pai provedor.

Além disso, confirmando parcialmente a hipótese inicial, extroversão e neuroticismo foram as únicas dimensões da personalidade paterna preditoras de gatekeeping materno, unicamente na dimensão de normas e responsabilidades. É importante notar que no relato das mães, quanto maior a extroversão paterna, menores foram os índices de gatekeeping materno relacionados às normas e responsabilidades. Em outras palavras, quanto mais sociáveis, enérgicos, ativos, expressivos e capazes de demonstrar emoções positivas os pais se mostraram, as mães perceberam que tendem a controlar menos o estabelecimento das normas, responsabilidades e os padrões de como as tarefas domésticas e de cuidado com as crianças devem ser desempenhadas.

Este resultado é importante, pois enfatiza a importância dos aspectos positivos da personalidade paterna que contribuem para um menor $g a$ tekeeping materno, e consequentemente menores 
atravessamentos maternos no envolvimento do pai com a criança, fator esse preditor de diferentes esferas do desenvolvimento infantil bem consolidado na literatura (Volling \& Cabrera, 2019). Esse resultado pode estar relacionado à capacidade paterna de expressão de suas ideias de forma mais assertiva, o que pode contribuir para a segurança materna na capacidade do pai para dividir as responsabilidades domésticas e também exerce sua parentalidade. Ademais, a diminuição das atitudes maternas relacionadas ao controle das normas e responsabilidade frente à extroversão paterna pode estar atrelada a uma interiorização por parte das mães do pressuposto cultural de gênero (Palkovitz et al., 2014) que atribui aos homens a responsabilidade parental pela brincadeira intensa, agitada ao mesmo tempo em que dão suporte emocional e impõem limites à criança (Bossardi et al., 2013). Isso porque a ideia de que pais extrovertidos são sociáveis, enérgicos e expressivos e desempenhariam de maneira satisfatória esta responsabilidade é culturalmente reconhecida como papel paterno e não materno. Assim, além de não ameaçar retirar da mãe responsabilidades culturalmente reconhecidas como maternas, estaria também contribuindo para uma visão tradicional e culturalmente aceita desta família.

Destacou-se também que na percepção dos pais, quanto maior o neuroticismo paterno, maiores foram os índices relatados em normas e responsabilidades. Ou seja, quando apresentaram como características individuais nervosismo, tensão, preocupação, irritação e instabilidade emocional, mais os próprios pais perceberam que as mães tendem a controlar as normas, responsabilidades e padrões de cuidado doméstico e de criação das crianças. Esses resultados distanciam-se de aspectos que podem influenciar positivamente a relação do pai com a criança quando relacionados aos baixos índices de neuroticismo. Prinzie et al. (2009) destacaram que a maior estabilidade emocional pode afetar positivamente a parentalidade por facilitar interações positivas entre pais e filhos/ as, e pode expandir as habilidades parentais em responder de forma apropriada os sinais e comportamentos das crianças.

No estudo de Schoppe-Sullivan et al. (2015) um funcionamento psicológico empobrecido caracterizado por aspectos negativos como neuroticismo, ansiedade e depressão, foi preditor de maiores relatos de gate closing pelas mães. A consonância entre os resultados de Schoppe-Sullivan et al. (2015) com os resultados da presente pesquisa parece ressaltar o papel protetivo do gatekeeping materno (Allen \& Hawkins, 1999). Isso porque pais com maiores traços de neuroticismo tendem a desempenhar uma parentalidade mais insegura, inibida, de pouca interação com a criança e negligência, cujo humor e comportamentos parentais são afetados negativamente, levando a uma parentalidade autoritária, rígida e coercitiva (Silva \& Vieira, 2018). Neste caso, o controle materno das normas e responsabilidades pelas tarefas domésticas e pela criação das crianças pode vir a proteger a criança de comportamentos negligentes, autoritários ou coercitivos exercidos pelos pais, indicados pela literatura como nocivos ao desenvolvimento infantil (Volling \& Cabrera, 2019).

\section{Considerações finais}

Ressalta-se a importância do estudo da relação entre a personalidade paterna e o gatekeeping materno, por englobar aspectos relacionados às características pessoais em interação com construções históricas e sociais de gênero que influenciam as crenças e comportamentos maternos de regulação do envolvimento paterno nas tarefas domésticas e cuidados com os filhos, e consequentemente, na qualidade e proximidade da relação pai-filho/a. Este estudo permitiu evidenciar que, de fato, o gatekeeping materno está presente nesta amostra de forma moderada, e que ele tem relações com a personalidade do pai. Permanece ainda a questão sobre como esses construtos se relacionam com a 
personalidade materna. Pais e mães parecem perceber que as mães exercem um controle sobre o envolvimento paterno com as tarefas domésticas e cuidados com os filhos que não são completamente inibitórios dessa aproximação, mas que também não colabora ativamente para que ela ocorra, ainda que os índices sejam de moderados para baixos.

Destaca-se a relevância de intensificar os estudos relacionados ao gatekeeping materno correlacionando-o com outras variáveis individuais dos pais, das mães ou das crianças, que podem influenciar tanto no tipo e frequência de gatekeeping materno, quanto nas características individuais de cada sujeito, como por exemplo, autoestima, temperamento e crenças sobre papéis sociais. Algumas limitações deste estudo são interessantes de serem mencionadas, como o fato de questões relacionadas aos relacionamentos conjugais e coparentais, satisfação e envolvimento paterno, também não terem sido avaliadas, sendo importantes para uma compreensão aprofundada da relação entre gatekeeping materno e personalidade do pai. Nesse sentido, também é indicado que pesquisas futuras sejam feitas a partir das diversas configurações familiares, do desenvolvimento típico ou atípico das crianças, e nos mais variados contextos de envolvimento paterno e desenvolvimento infantil.

Uma das maiores contribuições deste estudo é o fato de ter dado a possibilidade de os pais responderem por si mesmos sobre um construto que afeta diretamente a relação pai-filho, em sua maioria acessadas pelas pesquisas somente por intermédio da mãe. Isso permite um maior aprofundamento do fenômeno, pois traz à tona as percepções paternas sobre o gatekeeping materno, geralmente relacionado a como as próprias mães percebem os pressupostos culturais de gênero atribuídos às mulheres no encargo das funções relativas ao espaço doméstico e cuidado das crianças, mas que afeta diretamente os modos de ser pai. Por fim, merece destaque o fato de ser um dos primeiros estudos nacionais a trazer à tona a temática do $g a$ tekeeping materno relacionada às características individuais do pai, possibilitando o início de uma série de pesquisas, diálogos e reflexões sobre um tema com grande influência no estudo do envolvimento paterno, principalmente tendo em vista o crescimento constante de pesquisas que apontam para a importância do papel do pai no desenvolvimento infantil.

Ressalta-se que a participação na pesquisa gerou um efeito terapêutico nos respondentes. Alguns participantes relataram que a pesquisa permitiu reflexões sobre suas características pessoais ou do cônjuge que antes não eram pensadas, de forma que pretendiam conversar mais com os parceiros sobre o tema, com o intuito de se autoavaliarem e repensarem suas práticas buscando um aprimoramento para estabelecer cada vez mais um ambiente saudável de crescimento para os filhos. Deste modo, enfatiza-se a importância de se fomentar as pesquisas relacionadas à divisão de tarefas familiares, envolvimento parental e desenvolvimento infantil, bem como de espaços que promovam reflexões acerca do quanto características pessoais dos pais se manifestam na criação dos filhos e como elas podem influenciar o ambiente de desenvolvimento da criança.

\section{Referências}

Allen, S. M., \& Hawkins, A. J. (1999). Maternal Gatekeeping: Mothers' beliefs and behaviors that inhibit greater father involvement in family work. Journal of Marriage and the Family, 61, 199-212. https://doi.org/10.2307/353894

Andrade, J. M. (2008). Evidência de validade do inventário dos cinco grandes fatores de personalidade no Brasil [Tese de Doutorado, Universidade de Brasília]. https://repositorio.unb. br/handle/10482/1751

Bossardi, C. N., Gomes, L. B., Vieira, M. L., \& Crepaldi, M. A. (2013). Engajamento paterno no cuidado a crianças de 4 a 6 anos. Psicologia Argumento, 31, 237-246. http://www2.pucpr. 
$\mathrm{br} / \mathrm{reol} / \mathrm{pb} /$ index.php/pa?dd $1=7831 \& \mathrm{dd} 99=-$ view\&dd98=pb

Cabrera, N. J., \& Volling, B. L. (2019). Moving research on fathering and children's development forward: Priorities and recommendations for the future. In B. L. Volling \& N. J. Cabrera (Eds.), Advancing research and measurement on fathering and children's development. Monographs of the Society of Research in Child Development, 84(1), 107-117. https://srcd.onlinelibrary.wiley. com/doi/10.1111/mono.12404

Cannon, E. A., Schoppe-Sullivan, S. J., Mangelsdorf, S. C., Brown, G. L., \& Sokolowski, M. S. (2008). Parent characteristics as antecedents of maternal gatekeeping and fathering behavior. Family Process, 47, 501-519. https://doi.org/10.1111/j.15455300.2008.00268.x

De Young, C. G., Quilty, L. C., \& Peterson, J. B. (2007). Between facets and domains: Ten aspects of the Big Five. Journal of Personality and Social Psychology, 93, 880-896. https:// doi.org/10.1037/0022-3514.93.5.880

Fagan, J., \& Cherson, M. (2017). Maternal Gatekeeping: The Associations Among Facilitation, Encouragement, and Low-Income Fathers' Engagement With Young Children. Journal of Family Issues, 38(5), 633-653. https://doi. org/10.1177/0192513X15578007

Fagan, J. (2020). Broadening the scope of father-child attachment research to include the family context. Attachment \& Human Development, 22(1), 139-142. https://doi.org/10.1080/14616734.2 019.1589071

Frosch, C. A., Schoppe-Sullivan, S. J., \& O'Banion, D. D. (2021). Parenting and child development: A relational health perspective. American Journal of Lifestyle Medicine, 15(1), 45-59. https:// doi.org/10.1177/1559827619849028

Hauser, O. (2012). Pushing daddy away? A qualitative study of maternal gatekeeping. Qualitative Sociology Review, 8(1), 34-59. http://www.qualitativesociologyreview.org/ENG/Volume21/ QSR_8_1_Hauser.pdf
Holmes, E., Dunn, K. C., Harper, J., Dyer, W., \& Day, R. D. (2013). Mother knows best? Inhibitory maternal gatekeeping, psychological control, and the mother-adolescent relationship. Journal of Adolescence, 36, 91-101. https://doi. org/10.1016/j.adolescence.2012.09.010

John, O. P., Donahue, E. M., \& Kentle, R. L. (1991). The Big Five Inventory - Versions $4 a$ and 54. University of California; Institute of Personality and Social Research. https://www.ocf.berkeley. edu/ johnlab/bfi.htm

Lee, M. (2010). The big five traits and maternal gatekeeping at the transitions to parenthood [Senior Honors Thesis, The Ohio State University]. http://hdl.handle.net/1811/45471

Lee, J-K., Schoppe-Sullivan, S. J., Feng, X., Gerhardt, M. L., \& Kamp Dush, C. M. (2019). Longitudinal measurement invariance across fathers' and mothers' reports of maternal gatekeeping behavior. In B. L. Volling \& N. J. Cabrera (Eds.), Advancing research and measurement on fathering and children's development. Monographs of the Society of Research in Child Development, 84(1), 35-49. https://srcd.onlinelibrary.wiley. com/doi/10.1111/mono.12404

McCrae, R. R., \& John, O. P. (1992). An introduction to the five-factor model and its applications. Journal of Personality, 60(2), 175-215. http:// doi.org/10.1111/j.1467-6494.1992.tb00970.x

Nogueira, M. C. (2001). Feminismo e discurso do gênero na psicologia social. Psicologia \& Sociedade, 13(1), 107-128. http://hdl.handle. net/1822/4117

Palkovitz, R., Trask, B. S., \& Adamsons, K. (2014). Essential Differences in the Meaning and Processes of Mothering and Fathering: Family Systems, Feminist and Qualitative Perspectives. Journal of Family Theory \& Review, 6(4), 406-420. https://doi.org/10.1111/jftr.12048

Piskernik, B., \& Ahnert, L. (2019). What does it mean when fathers are involved in parenting? In B. L. Volling \& N. J. Cabrera (Eds.), Advancing research and measurement on fathering and 
children's development. Monographs of the Society of Research in Child Development, 84(1), 64-78. https://srcd.onlinelibrary.wiley. com/doi/10.1111/mono.12404

Prinzie, P., Stams, G. J. J. M., Deković, M., Reijntjes, A. H. A., \& Belsky, J. (2009). The relations between parents' Big Five personality factors and parenting: A meta-analytic review. Journal of Personality and Social Psychology, 97(2), 351-362. https://doi.org/10.1037/a0015823

Puhlman, D. J., \& Pasley, K. (2017). The Maternal Gatekeeping Scale: Constructing a Measure. Family Relations, 66(5), 824-838. https://doi. org/10.1111/fare.12287

Rosseel, Y. (2012). lavaan: An R Package for Structural Equation Modelin. Journal of Statistical Software, 48(2), 1-36. http://www.jstatsoft.org/v48/i02/

Schoppe-Sullivan, S. J., Altenburger, L. E., Lee, M. A., Bower, D. J., \& Kamp Dush, C. M. (2015). Who are the gatekeepers? Predictors of maternal gatekeeping. Parenting: Science and Practice, 15, 166-186. https://doi.org/10.1080/1529519 2.2015.1053321

Schoppe-Sullivan, S. J., Brown, G. L., Cannon, E. A., Mangelsdorf, S. C., \& Sokolowski, M. S. (2008). Maternal Gatekeeping, coparenting quality, and fathering behavior in families with infants. Jour- nal of Family Psychology, 22, 389-398. https:// doi.org/10.1037/0893-3200.22.3.389

Silva, M. L. I., \& Vieira, M. L. (2018). Relações entre a parentalidade e a personalidade de pais e mães: uma revisão integrativa da literatura. Estudos e Pesquisas em Psicologia, 18(1), 361383. https://doi.org/10.12957/epp.2018.38125

Sweeney, K. K., Goldberg, A. E., \& Garcia, R. L. (2017). Not a "mom thing": Predictors of gatekeeping in same-sex and heterosexual parent families. Journal of Family Psychology, 31(5), 521-531. https://doi.org/10.1037/fam0000261

Volling, B. L., \& Cabrera, N. J. (2019). Advancing research and measurement on fathering and child development: Introducing the issues and a conceptual framework. In B. L. Volling \& N. J. Cabrera (Eds.), Advancing research and measurement on fathering and children's development. Monographs of the Society of Research in Child Development, 84(1), 7-17. https://doi. org/10.1002/mono.12404

Zvara, B. J., Schoppe-Sullivan, S. J., \& Dush, C. (2013). Fathers' involvement in child health care: Associations with prenatal involvement, parents' beliefs, and maternal gatekeeping. Family Relations, 62, 649-661. https://doi.org/10.1111/ fare. 12023

\section{Recebido: janeiro 11, 2018 Aprovado: julho 2, 2021}

